\title{
Archéopages
}

Archéopages

Archéologie et société

Hors-série 3 | 2012

Nouveaux champs de la recherche archéologique

\section{De la contribution à la synergie : une décennie de relations archéologues-géomorphologues à l'Inrap}

Laurent Bruxelles

\section{CpenEdition}

1 Journals

Édition électronique

URL : https://journals.openedition.org/archeopages/446

DOI : 10.4000/archeopages.446

ISSN : 2269-9872

Éditeur

INRAP - Institut national de recherches archéologiques préventives

Édition imprimée

Date de publication : 1 janvier 2012

Pagination : $21-24$

ISSN : $1622-8545$

\section{Référence électronique}

Laurent Bruxelles, « De la contribution à la synergie : une décennie de relations archéologuesgéomorphologues à l'Inrap », Archéopages [En ligne], Hors-série 3 | 2012, mis en ligne le 01 janvier 2012, consulté le 25 février 2023. URL : http://journals.openedition.org/archeopages/446 ; DOI : https://doi.org/10.4000/archeopages.446 


\section{De la contribution à la synergie: une décennie de relations archéologues-géomorphologues à l'Inrap}

Laurent Bruxelles

Inrap, UMR 5608 «Travaux et Recherches Archéologiques sur les

Cultures, les Espaces et les Sociétés », Université de Johannesburg

D ans l'histoire de l'archéologie, l'utilisation des compétences de spécialistes des sciences de la terre est apparue très rapidement comme une nécessité. Ce constat est encore plus vrai pour les périodes anciennes de la Préhistoire, où le facteur géologique devient primordial si l'on veut connaitre les environnements contemporains de l'occupation, mais aussi l'histoire des sites. Pour les périodes historiques, ce besoin semblait moins évident et, même aujourd'hui, le recours à un géomorphologue n'est pas systématique. Pourtant, quelle que soit la période concernée, le géomorphologue, par son regard extérieur, apporte toujours des éléments de réflexion nouveaux et contribue à l'exploitation ainsi qu'à la mise en perspective des données issues de la fouille.

Une participation devenue de plus en plus systématique. Dès l'Afan, une équipe de géomorphologues et de pédologues a assuré les études géoarchéologiques de certaines opérations d'archéologie préventive. Quelques CDD, voire des contrats privés, permettaient de renforcer ponctuellement les effectifs et de suivre les chantiers qui le nécessitaient le plus. Bien souvent, faute de moyens ou de personnel, mais peut-être surtout parce que cette pratique n'était pas encore très courante, nombre d'opérations n'ont pourtant pas été suivies par ces spécialistes. En outre, lorsque des renforts étaient demandés, les interventions avaient lieu le plus souvent au cours des fouilles, alors qu'il n'y avait pas eu de suivi géomorphologique lors du diagnostic. Or c'est cette phase de détection des sites qui permet de collecter la plus grande quantité de données. De ce fait, l'étude d'un site en cours de fouille pouvait parfois donner l'impression de regarder "par le petit trou de la lorgnette», privant les chercheurs d'informations complémentaires qui auraient pu être apportées au moment du diagnostic. Il manquait une échelle intermédiaire entre le paysage et le site, celle que couvre bien souvent l'emprise du diagnostic.

Avec la création de l'Inrap ce type de collaboration a progressivement été systématisé dès le diagnostic. Fort des expériences passées et des besoins grandissants, des postes de géoarchéologues ont été pourvus lors des plans de recrutement successifs, si bien que chaque interrégion (il demeure néanmoins une exception à ce jour), dispose d'un ou de plusieurs géoarchéologues, toutes spécialités confondues. Au sein de nos interrégions, qui ne sont pas imperméables au demeurant, les opérations sont réparties soit de manière géographique, soit suivant les thématiques géologiques ou chronologiques selon les spécialités des uns et des autres. L'objectif étant d'assurer le suivi le plus complet possible de toutes les opérations requérant l'intervention d'un géomorphologue. Le plus difficile restant de concilier, tout au long de l'année, les impératifs de présence sur le terrain et les délais de rendu d'un nombre grandissant de rapports...

Un apprivoisement progressif. L'un des grands apports de l'intervention de spécialistes au cours d'une opération archéologique reste la plus-value scientifique conséquente à l'interdisciplinarité. Il faut d'ailleurs préciser que les géomorphologues n'ont de « spécialistes » que le nom, en tant que professionnels d'une discipline annexe à l'étude archéologique. Mais, à l'Inrap, la plupart sont de vrais généralistes, capables d'intervenir dans des domaines variés et sur toutes les périodes ou presque! En plus de cette polyvalence, certains d'entre nous ont développé des spécialités qui les ont fait reconnaître par la communauté scientifique et, ce qui n'est pas négligeable, par leurs pairs. Au fil des années, les archéologues et les géomorphologues se sont peu à peu apprivoisés : pour les archéologues, il a fallu s'habituer au vocabulaire parfois quelque peu alambiqué des géologues, alors que ces derniers ont dû intégrer les problématiques archéologiques qui imposent des échelles chronologiques et géographiques assez déroutantes au début. Peu à peu, le géomorphologue est devenu géoarchéologue et un véritable dialogue constructif s'est établi. L’archéologue est lui aussi devenu familier de la géomorphologie, identifiant par lui-même certains phénomènes avant le passage du spécialiste! Les discussions sur le terrain, la rédaction en commun des chapitres de synthèse, les publications, mais aussi les actions de recherche et de formation au sein de notre institut sont autant de points forts qui favorisent ce dialogue constructif.

Cette interdisciplinarité ressort de plus en plus dans les très nombreux rapports produits annuellement par l'Inrap. Même si la progression n'est pas uniforme, espérons que le temps est loin où nos contributions étaient reléguées dans les annexes du rapport, sans aucune exploitation, ou presque, de ses apports pour l'interprétation des faits ou la synthèse. Dans certain cas, notre participation purement formelle était considérée au mieux comme une "caution scientifique ", si tant est qu'une caution soit nécessaire.

L'intégration croissante de la géomorphologie dans l'archéologie préventive. Peu à peu, l'habitude de travailler de concert avec des géomorphologues a été prise. La plupart du temps, les responsables d'opération ont contacté directement les géomorphologues. Ils le font soit un peu avant le début d'un chantier, soit lorsqu'un questionnement apparait. À nous d'essayer de répondre rapidement, tant que les coupes sont visibles... D'autres collègues se sont mis à solliciter le géomorphologue quasi systématiquement, par intérêt scientifique ou parce qu'ils savent que les terrains qu'ils vont recouper nous intéressent, que ce soit du point de vue géologique (terrasses alluviales, littoral, karst...) ou chronologique (Pléistocène, Holocène, 


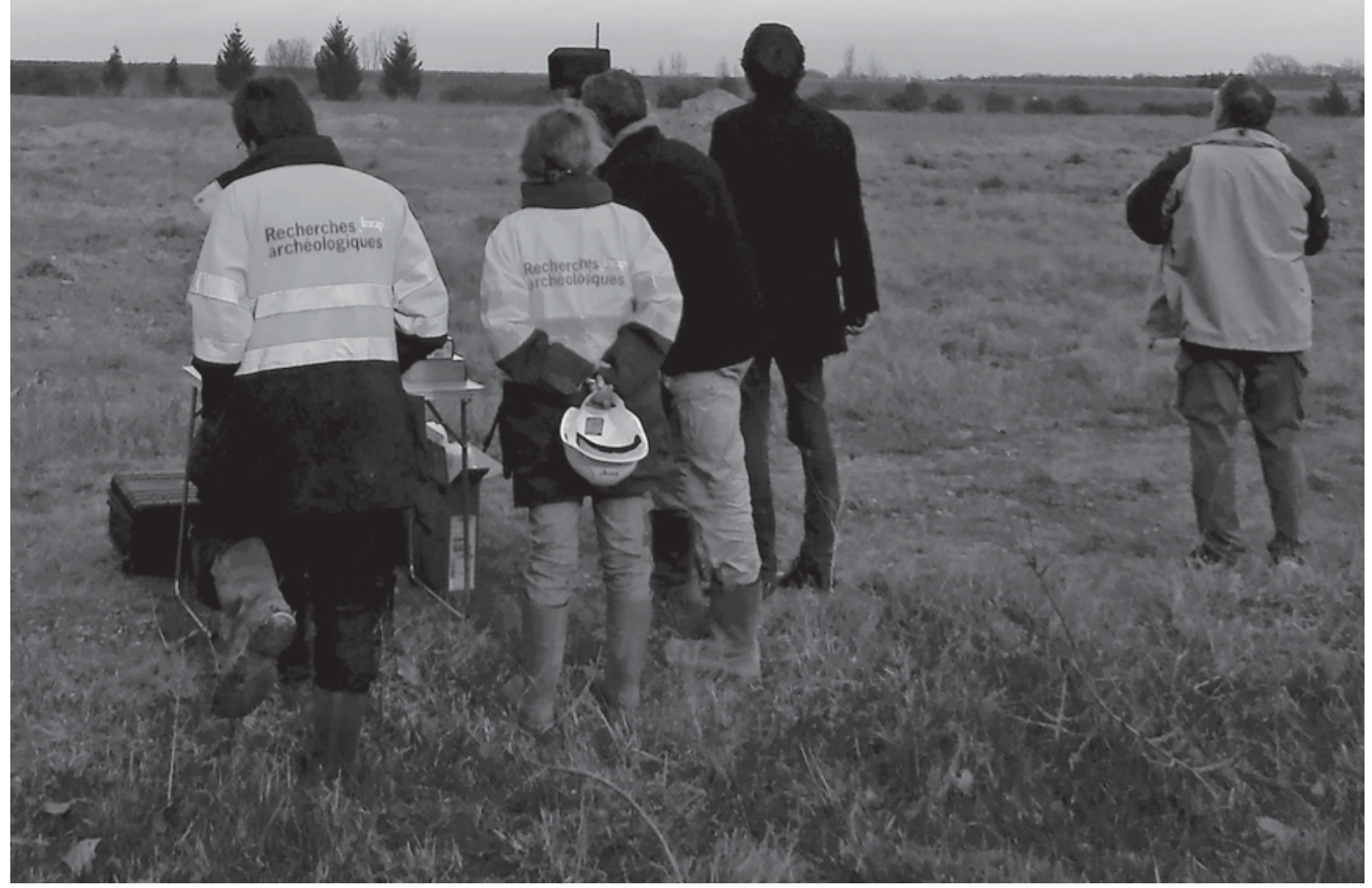

[Fig.1] Première expérience de repérage de structures archéologiques par un drone équipé d'une caméra

thermique (les Ménudes, Colomiers,

Haute-Garonne; responsable

d'opération: Marc Jarry ; programme

Archéodrones sous la direction de

Florent Hautefeuille, UMR 5608

«Travaux et Recherches

Archéologiques sur les Cultures,

les Espaces et les Sociétés»). 
périodes historiques...). Quelle que soit la motivation qui est à l'origine de notre intervention sur le terrain, il en découle toujours un enrichissement du corpus des données qui, tôt ou tard, bénéficie à l'archéologie préventive.

Certaines questions reviennent fréquemment dans nos enquêtes : à quelle profondeur se trouve le «substrat géologique» et comment le reconnaître afin d'être sûr d'avoir traversé tous les terrains susceptibles de contenir de la matière archéologique? Comment établir une lecture stratigraphique complète, faisant la part de ce qui relève des formations sédimentaires, des horizons pédologiques et des perturbations anthropiques? De quelle façon déterminer si une structure douteuse correspond à un fait archéologique ou à un phénomène naturel? Ces données «opérationnelles», complétées par toutes les autres informations que nous sommes susceptibles de relever, sont ensuite remises en perspective à l'échelle du paysage et de l'histoire géomorphologique régionale. Et, hasard ou chance de l'archéologie préventive, nous avons souvent l'occasion de réaliser des observations là où personne n'avait eu la possibilité ni même l'idée d'aller les chercher jusque-là. Ces données nouvelles et les interprétations que nous en tirons contribuent toujours à accroître, voire à renouveler, la connaissance sur l'histoire des paysages et des hommes. Il est alors possible, avec toute la prudence que requiert ce type d'exercice, de parler de potentialité pour certaines périodes, certains types d'occupation ou certains contextes géomorphologiques.

Au cours de l'opération, le géomorphologue est souvent le premier spécialiste à intervenir sur le terrain ; c'est même souvent le seul à suivre l'opération d'un bout à l'autre et donc d'avoir une vision globale des problématiques. il constitue donc un maillon important en tant que relai vers les autres spécialistes, en conseillant le responsable d'opération en fonction de la période et du milieu concernés, mais aussi des conditions de conservation. Cette coordination paléoenvironnementale, plus ou moins improvisée, constitue même un poste central dans certaines grandes opérations.

Mais, au-delà de cette relation scientifique et humaine qui constitue la base de nos interventions, les géomorphologues sont de plus en plus sollicités pour intervenir à d'autres niveaux dans la réalisation d'une opération d'archéologie préventive. Si la participation d'un géomorphologue est très souvent requise dès la prescription, il arrive parfois que certaines opérations ne soient prescrites quà finalité paléoenvironnementale. Dans des contextes mal connus ou pour des périodes non documentées, ce type d'intervention est à même d'éclairer certains aspects précis de l'histoire des paysages, de l'origine des formations superficielles ou d'exploiter des données paléoenvironnementales inédites. Sur ces bases, il est parfois possible d'avoir une idée du potentiel archéologique et des moyens qu'il faudra mobiliser pour les rechercher. Pendant la préparation de l'opération, le géomorphologue peut également être d'un grand secours. En fonction des contextes, il peut indiquer jusqu'à quelle profondeur on pourra espérer trouver des vestiges, ce qui aura un impact direct sur le volume de moyens à mettre en ouvre. De même, il pourra renseigner sur le type de sondages à prévoir, mais aussi sur leur fréquence et leur disposition. D’ailleurs, à ce titre, plusieurs expériences de prospections préalables ont été réalisées avec succès. Lorsque le contexte morphologique et sédimentaire le permet, une équipe constituée d'un archéologue et d'un géomorphologue reconnaît en détail l'ensemble de l'emprise concernée. Puis, en fonction des observations, une cartographie de l'intervention est établie de concert, délaissant certaines parties jugées stériles (substrat affleurant par exemple) au profit de secteurs à plus forte potentialité. L'opération peut alors être organisée sur cette base rationnelle.

En parallèle, des outils complémentaires de plus en plus performants sont exploités par les géomorphologues. Outres les documents topographiques et géologiques classiques, les sondages géotechniques réalisés souvent avant notre intervention sont des données disponibles qui peuvent être très utiles. Ils peuvent être complétés par ceux consultables en ligne depuis quelques années et rassemblés dans la Banque de Données du Sous-Sol du BRGM. Par ailleurs, les modèles numériques de terrain (BD Alti de l'IGN ou Lidar) constituent un véritable outil $3 \mathrm{D}$, à la fois pour repérer ou interpréter des formes de la surface du sol, mais aussi pour produire des illustrations pédagogiques et convaincantes. Plus ponctuellement, les données géophysiques, encore relativement coûteuses, sont utilisées pour répondre à des questions précises : recherche de bâtis, profondeurs de puits, présence de cavités... Enfin, des tests de repérage par drones sont en cours de réalisation sur des opérations de Grand Sud-Ouest et de Méditerranée [Fig.1]. Équipés d'une caméra thermique à haute sensibilité, ils sont capables de détecter en temps réel certaines anomalies telles que des fosses ou des fossés, du bâti ou des cavités invisibles en surface.

\section{Vers une véritable synergie scientifique?}

L'archéologie préventive permet d'investir des domaines très diversifiés et parfois peu connus. Sur de grandes surfaces, on obtient une information à haute résolution qu'aucune autre méthode de recherche géologique ne permet : ouverture entre 5 et 10\% de la surface, sondages profonds et continus, relevés de logs stratigraphiques in situ, prélèvements d'échantillons, topographie détaillée permettant d'établir des relations géométriques entre chaque formation sédimentaire sur l'ensemble de la zone étudiée [Fig.2]. Des modèles numériques de terrain (MNT) peuvent même être construits à partir de ces données afin d'interpréter et d'illustrer la géométrie de certaines couches repères, comme le toit de la terrasse alluviale, les paléosols ou des niveaux de troncature. Dès la phase de terrain, toute une série d'autres spécialistes sont susceptibles d'apporter leur contribution en fonction des découvertes. Enfin, le travail aux côtés d'archéologues donne la mesure du facteur anthropique qui, même en contexte « hors site » 

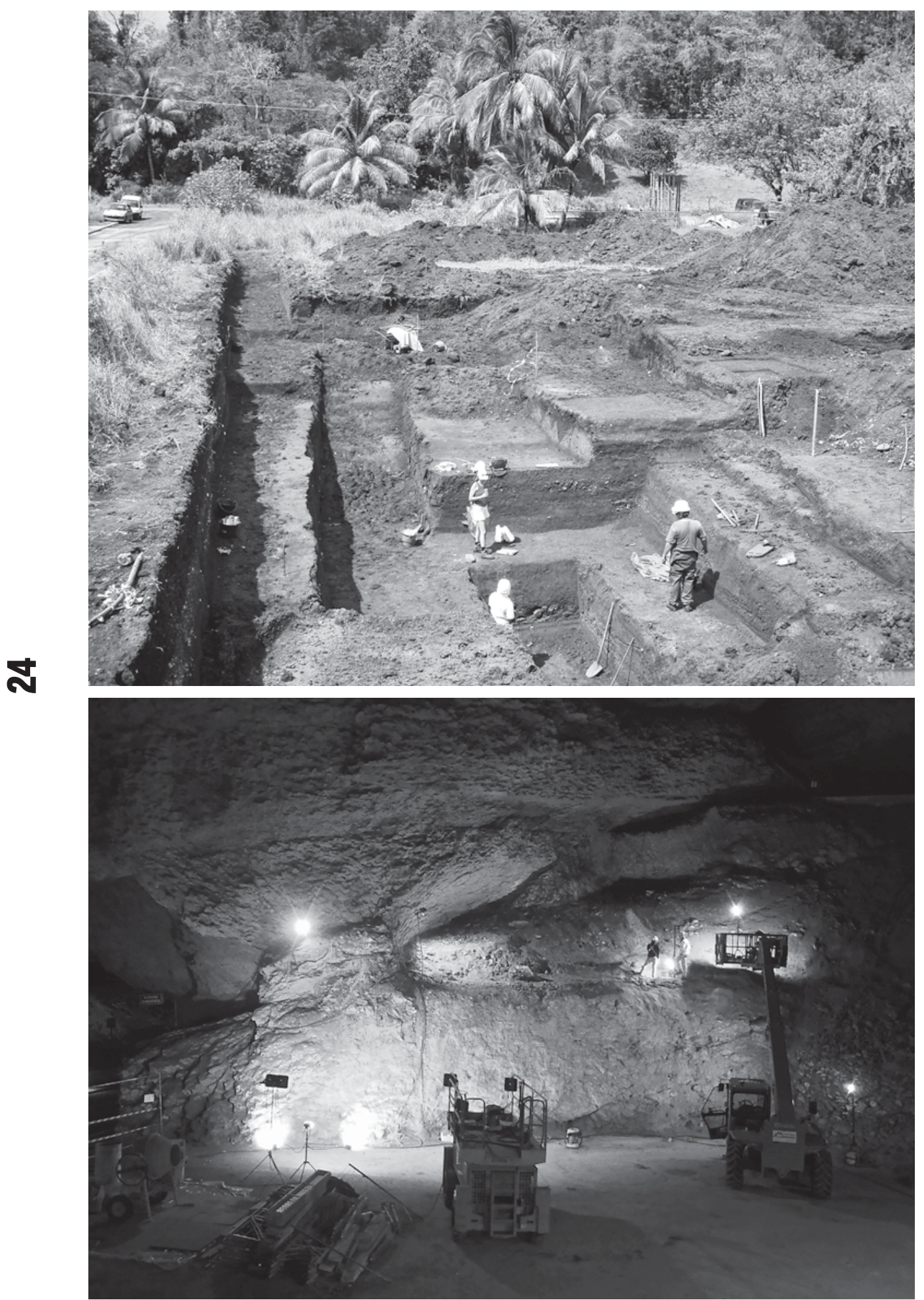

[Fig.2] Lors de la fouille du site de Yuikét (Gourbeyre, Guadeloupe; responsable d'opération : Dominique Bonnissent) une partie de l'emprise a été réservée spécifiquement à la réalisation d'un tranchée profonde afin de collecter toutes les informations paléoenvironnementales associées ou non aux paléosols amérindiens.
[Fig.3] Diagnostic archéologique réalisé fin 2011 dans la grotte du Mas d'Azil (Campagne-sur-Arize, Ariège ;

responsable d'opération : Marc Jarry). La découverte de nouveaux vestiges, mais aussi de formations sédimentaires clés renouvelle complètement

les connaissances de cette grotte ornée et des différentes occupations fouillées de longue date. Sur cette base, et en appui à des études archéologiques en cours (responsable Carole Fritz, UMR 5608 « Travaux et Recherches Archéologiques sur les Cultures, les Espaces et les Sociétés »),

un nouveau programme scientifique dirigé par des agents de l'Inrap vient d'être lancé. 
est bien souvent sous-évalué dans les schémas classiques d'évolution du paysage.

L'ensemble des observations collectées constitue donc un enregistrement riche, de grande qualité et bien souvent inédit. Ce travail prend encore plus d'intérêt dans le cadre de vastes superficies, mais aussi de tracés de grands linéaires où de véritables transects peuvent êtres dressés sur plusieurs dizaines, voire plusieurs centaines de kilomètres de longueur. C'est également le cas pour des zones à forte urbanisation que nous investissons peu à peu au fil des années et où les données concernent de très grandes surfaces. Reste ensuite à réussir à dresser des synthèses géomorphologiques et paléoenvironnementales à partir de cette masse d'informations, entre autres par l'usage de bases de données et de SIG.

Les résultats sont autant d'acquis pour les opérations de diagnostic à venir puisque, peu à peu, l'ensemble des contextes géomorphologiques de chaque région se trouve de mieux en mieux caractérisé. De même, lors d'une fouille, la maîtrise de l'ensemble du contexte environnemental permet d'emblée de replacer la ou les occupations dans leur cadre initial, depuis le site jusqu'au paysage. De ce voyage commun depuis dix ans commence donc à émerger une nouvelle dimension dans l'interprétation de nos résultats archéologiques et géomorphologiques. L'expérience, mais aussi le volume de données disponibles, permettent d'aller de plus en plus loin dans les interprétations, une discipline éclairant l'autre et vice-versa.

Conséquence directe de cette évolution, des monographies et des articles de synthèses commencent à être régulièrement publiés et de plus en plus souvent dans de prestigieuses revues internationales. À chaque fois, ils sont le résultat de cet apport mutuel tout en restant une étape de plus dans l'évolution des connaissances scientifiques.

Comme on s'amuse souvent à le dire entre nous, certes avec un manque d'humilité flagrant, en dix ans de suivi géomorphologique à l'Inrap, nous avons étudié plus de coupes que tous nos professeurs de géologie réunis ! Cette grande expérience du terrain, doublée d'un réseau fonctionnel de spécialistes et d'une intégration complète dans le domaine de la recherche aboutit à la formation de véritables experts aussi à l'aise sur le terrain qu'au laboratoire ou devant l'ordinateur. Cette aptitude, qui caractérise de plus en plus une grande partie des agents de terrain est largement reconnue et déborde du seul cadre de notre institut. Comme bon nombre d'agents de l'Inrap en général, nous sommes très régulièrement sollicités pour participer à des fouilles programmées, pour intervenir à l'université ou pour exporter nos compétences à travers le monde. De prestataires, nous sommes devenus des partenaires et désormais, de plus en plus souvent, des responsables de projets scientifiques [Fig.3].

L'Inrap, par la surface de terrain sondée ou fouillée chaque année, mais aussi par les moyens mis en œuvre pour permettre de les étudier, a largement contribué au renouvellement des connaissances concernant les formations superficielles et la dynamique des paysages. Des organismes comme le BRGM sont d'ailleurs très demandeurs d'une convention d'échange de données avec notre institut.

Enfin, une véritable synergie apparaît peu à peu entre les géomorphologues et les archéologues. Ce que l'on appelle finalement l'interdisciplinarité renvoie ici à une parfaite complémentarité qui grandit au fur et à mesure que nous travaillons ensemble et que nous nous comprenons. Même les espaces vides de vestiges archéologiques se remplissent de sens et rendent positif des diagnostics qui étaient restés jusque-là négatifs. Si l'on arrive aujourd'hui à ce résultat, c'est aussi peut-être parce que les géomorphologues et les archéologues sont faits pour se comprendre : ce sont des passionnés d'histoire, que ce soit celle de la Terre ou celle des Hommes.

\section{Anthropologie biologique et archéologie : regards croisés?}

Hervé Guy

Inrap, Centre Norbert Elias

Anne Richier

Inrap, UMR6578 «Anthropologie bioculturelle »

L es disciplines scientifiques ont de tout temps puisé dans les appareils conceptuels et méthodologiques, ainsi que dans les fondements heuristiques de leurs voisines. En conséquence, elles s'enrichissent mutuellement à travers ce qu'il est communément nommé la pluridisciplinarité ou la transversalité. Il apparaît nécessaire, pour bien mesurer les liens entre anthropologie et archéologie, d'opérer un bref retour sur l'histoire de la discipline.

Historiographie disciplinaire. L'anthropologie biologique trouve son acception moderne vers le milieu du $\mathrm{XIX}^{\mathrm{e}}$ siècle, sous l'impulsion de Paul Broca et d'Armand de Quatrefages, alors que Darwin vient de publier son monumental De l'origine des espèces et que son cousin Galton élabore ses théories eugénistes. Lorsque la Société d'Anthropologie est créée, elle inscrit dans ses statuts « l'étude des races humaines », recherche que l'on retrouve sous la mention «zoologie» dans les comptes rendus de l'Académie des sciences (Blanckaert, Ducros, Hublin, 1989). C'est dans ce contexte intellectuel que l'anthropologie se veut une science de l'histoire naturelle de l'homme. Ayant du mal à rompre avec la méthode classificatrice héritée de Linné et de Buffon, et s'insérant dans un contexte géopolitique dominé par l'expansion du monde western et la constitution des grands empires coloniaux, l'anthropologie biologique («physique», dit-on à cette époque) accouchera de théories racistes et sociologiques, très vite utilisées par le discours politique. La Shoah, à cet égard, restera l'exemplaire et morbide avatar de cette conception hiérarchisée de l'homme.

L'anthropologie, en tant que science naturelle, ainsi

(1)

